\title{
Artificial Intelligence Nose (Aino-Pro) for the Medical Field
}

\author{
Masatoshi Kusuhara, M.D. \& Deputy Director ${ }^{1}$, Hanzo Tsuzuki, Managing Director ${ }^{2}$ \\ 1 The Shizuoka Cancer Center Research Institute. \\ 1007, Shimonagakubo, Nagaizumi-cho, Sunto-gun, Shizuoka, 411-8777 Japan \\ 2 Tokai-Denshi Inc. \\ 247-15, Atsuhara, Fuji-shi, Shizuoka, 419-0201 Japan \\ Corresponding author's e-mail address, \\ h_tsuzuki@tokai-denshi.co.jp
}

\begin{abstract}
Treatment of odor in the medical field
In cancer specialty hospitals, odor (disease odor) in the final stages of cancer is a serious problem. We have developed a portable gas chromatograph for determining odor, and for use in the treatment of said odor. Moreover, by utilizing artificial intelligence, a system (Aino-Pro) which can mimic human olfaction was developed.
\end{abstract}

Key words: Al-nose, artificial intelligence, odor, portable gas chromatograph, cancer

\section{Introduction}

There are various odors in hospitals. In the case of cancer specialty hospitals, odors (disease odor) present at the final stages of cancer are a serious issue. Analyzing the cancerous odor of patients with breast cancer and uterine cancer, we have created a model odor, and developed a product for deodorization.

Even though it is said a bad smell (cancer odor) occurs at the end of cancer, the quality and intensity of odor varies depending on the type of cancer and the patient. In clinical practice, as part of the cancerous area's treatment, washing, disinfection, Mohs ointment, antibiotics and so on are often utilized in tandem. What treatment to take depends subjectively on the nurse's sense of smell. We thought that if we had a bedside odor diagnostic device, objective evaluation would be possible, and began developing such a machine.

Since most of the odorous components are composed of VOC gases (volatile organic compounds), we discovered through trials by analyzing the main components given off as odor and we have developed a simple gas chromatograph that is compact, portable. Our approach utilizes indoor air as a carrier gas, therefore making it possible to detect gas stably by combining a PID (photoionization detector) and a semiconductor gas sensor. Furthermore, by utilizing artificial intelligence, we can simultaneously mimic the ability to distinguish odor levels akin to human olfaction.

At first, our initial step was to use a wine sample aroma kit (The 54 Masterkit aromas) as a trial odor test, which demonstrated the device's ability to identify and recognize to some extent the various wine contents. Moving forward, we are confident that in the future we will exceed the human sense of smell by further improving device sensitivity and shortening the measurement time.

\section{Operating principles}

To begin with, humans have about 400 kinds of olfactory cells that can identify odor. At this time, we have developed a detection unit that replaces these olfactory cells as a portable gas chromatograph for odor determination. (Figure 1)

The carrier gas used indoor air for gas chromatography, and VOC gases contained therein are removed by a filter. A capillary column of $30 \mathrm{~m}$ was used for gas separation, and a semiconductor gas sensor complementing a PID sensor were used for gas detection.

This constitutes the detection sensor that replaces the 400 kinds of olfactory cells held by humans. However while human olfactory sense is inferior to that of say a dog, even a human sense of smell has the ability to detect extremely low concentrations of odor gas. For that reason, a TD tube was adopted as a concentration method for detecting low concentrations of gas. As a result, concentrations of about 100 to 200 times normal levels became possible, and the sensitivity limit of the sensor was relatively improved. 
In addition, human beings are able to know the information obtained by olfactory cells and the cerebrum through utilizing neural connections and therefore identifying what kind of odor it is. By utilizing information obtained by artificial intelligence on the software side of things, odor recognition can be mimicked by A.I. in the same way as human olfactory cells. Just like human beings, odors experienced for the first time are memorized as new data so that the A.I. system learns and grows each time.

After acquiring an odor, it is analyzed by the A.I. software and outputs results based on the learned odor data. Many of the normal odors in daily life are comprised of VOC gases, but odorless gases are not able to be detected by humans. However, the prime characteristic of an artificial intelligence nose is that it is possible to judge complicated odors caused by metabolic degradation of cells in the body which are undetectable by humans.

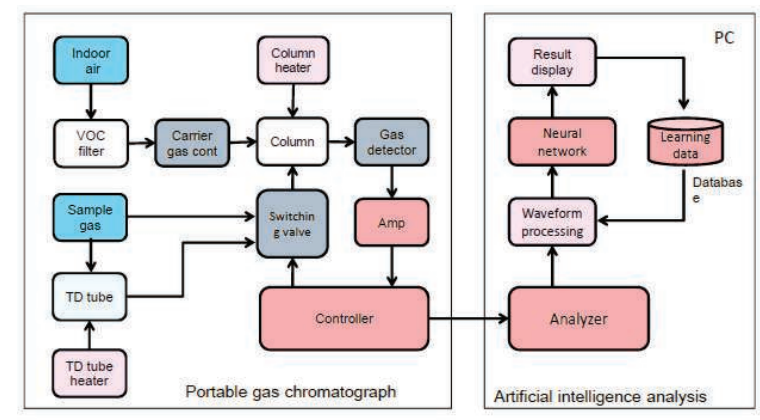

Figure 1, Portable gas chromatograph system

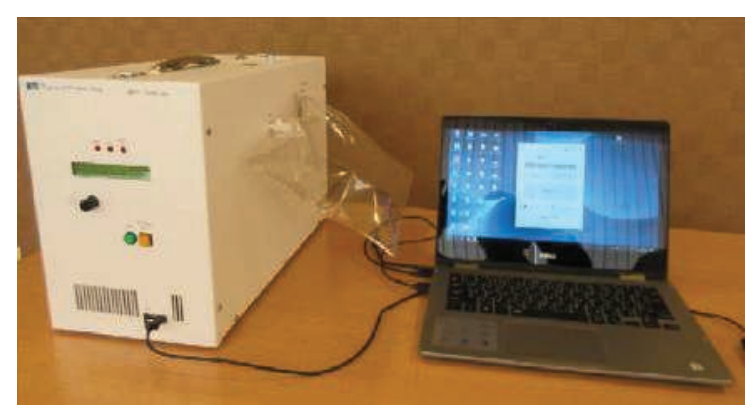

Photo 1, Aino-Pro System

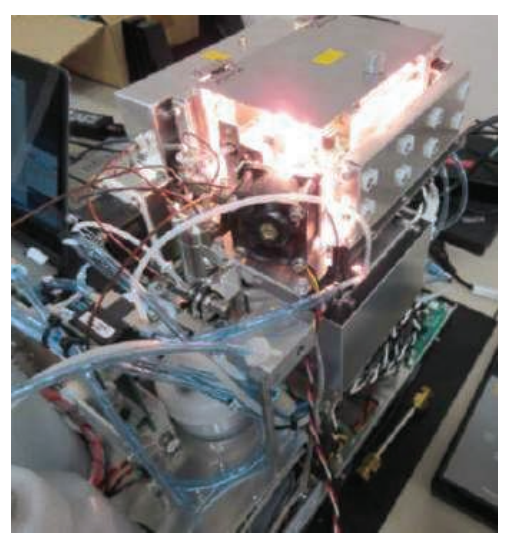

Photo 2, Internal view of Aino-Pro in operation

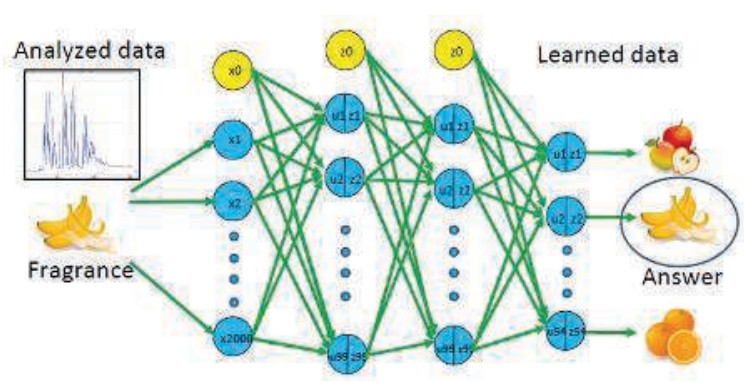

Figure 2, System of the Neural Network

\section{Issues and prospects}

Looking forward, in order to increase the odor determination ability, it is necessary to develop an ultra-sensitive VOC gas detection system and to shorten the measurement time.

From now, we are confident that additionally we can develop specialized odor detection devices for use in areas such as detecting odors from livestock farms, food processing, scent development of food samples, halitosis detection, drug doping etc.

\section{References}

[1] Fruit Photo. @copyright 2015 All-freedownload.com 